\title{
Awake cardiac surgery using a novel anesthetic technique
}

\section{[Une nouvelle technique anesthésique pour la chirurgie cardiaque vigile]}

Thomas M. Hemmerling md deaa, ${ }^{*}$ Nicolas Noiseux md frcsc msc, $†$ Fadi Basile md fresc,$\dagger$ Marie-Frédérique Noël md, $\dagger$ Ignatio Prieto md frcsc $†$

Purpose: We describe the first published cases of awake cardiac surgery in Canada. In addition, a novel anesthetic technique consisting of combined femoral block/high epidural thoracic anesthesia is presented.

Clinical features: Two patients, both $65 \mathrm{yr}$ of age and with good left ventricular function, were scheduled to undergo offpump coronary artery bypass grafting (OPCAB) for two grafts each. Anesthesia consisted of combined femoral 3:I block and high thoracic epidural anesthesia. Both surgeries proceeded without hemodynamic or respiratory complications; in both cases, opening of the pleural spaces was treated with insertion of thoracic drainage tubes. Both patients were transferred to the postanesthesia care unit immediately after surgery and six hours later to the cardiac surgical ward. Both patients were discharged from the hospital within five days of surgery.

Conclusion: We conclude that awake OPCAB is feasible using a combined femoral block/high thoracic epidural anesthesia technique which allows cardiac surgery and harvesting of the saphenous vein. Further clinical experience is required to define the technical limitations of this technique before randomized studies should be undertaken to better define the role of awake procedures in the future of cardiac surgery.

Objectif : Décrire les premiers cas publiés de cardiochirurgie vigile au Canada. De plus, présenter une nouvelle technique anesthésique qui est une combinaison de bloc fémoral et d'anesthésie péridurale thoracique haute.

Éléments cliniques: Deux patients de 65 ans, ayant une bonne fonction ventriculaire gauche, devaient subir un pontage aortocoronarien à cœur battant (PACCB), chacun pour une double greffe. L'anesthésie combinait un bloc fémoral 3:I et une anesthésie péridurale thoracique haute. Les opérations n'ont présenté aucune complication hémodynamique ou respiratoire; dans les deux cas, l'ouverture des espaces pleuraux a été traitée avec l'insertion de tubes de drainage thoracique. Les deux patients ont été transférés à la salle de réveil immédiatement après l'opération et six heures plus tard, à l'unité de chirurgie cardiaque. Les deux patients ont quitté l'hôpital dans les cinq jours après l'opération.

Conclusion: Le PACCB vigile peut se faire avec une technique qui combine le bloc fémoral et l'anesthésie péridurale thoracique haute, permettant la chirurgie cardiaque et le prélèvement de la veine saphène. L'expérience clinique doit s'étendre pour permettre de définir les limites techniques de cette méthode avant que des études randomisées soient entreprises pour préciser le rôle des opérations vigiles dans l'avenir de la cardiochirurgie.

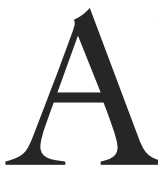

WAKE cardiac surgery can be performed under local or regional anesthesia. The introduction of less-invasive techniques to access the heart, such as endoscopic heart surgery or minimally invasive cardiac surgery in the form of mini-sternotomy or -thoracotomy have generated interest in different, less invasive strategies of cardiac anesthesia. In addition, the introduction of local or regional anesthetic techniques ${ }^{1,2}$ into the repertoire of cardiac anesthesia has enabled us to provide better postoperative analgesia, reduce the need for general anesthesia or use of opioids, whilst still providing good or even better surgical conditions.

Recently, cardiac surgery under regional anesthesia has been published, mainly in Europe with techniques based on the use of high thoracic epidural anesthesia (TEA). ${ }^{3-12}$ The (few) published cases are mostly associated with minimal invasive surgical techniques and a limitation of the number of grafts to avoid harvesting of the saphenous vein. Obviously, high thoracic anesthesia does not provide analgesia in the area where

From the PeriCARG (Perioperative Cardiac Research Group), Departments of Anesthesiology, ${ }^{*}$ and Cardiac Surgery, $†$ Centre Hospitalier de l'Université de Montréal (CHUM), Hôtel-Dieu, Université de Montréal, Montréal, Québec, Canada.

Address correspondence to: Dr. Thomas M. Hemmerling, Centre Hospitalier de l'Université de Montréal (CHUM), Hôtel-Dieu, Université de Montréal, 3840, rue Saint-Urbain, Montréal, Québec H2W 1T8, Canada. Phone: 514-890-8000, ext. 14570;

Fax: 514-412-7222; E-mail: thomashemmerling@hotmail.com

Accepted for publication June 9, 2005.

Revision accepted July 4, 2005. 
vein harvesting is usually performed, the thigh or calf. There is only one study where harvesting of the saphenous vein is performed in awake cardiac surgery, using low spinal anesthesia in addition to high thoracic anesthesia. ${ }^{11}$

We present a novel technique of 'femoral' 3:1 block and high thoracic epidural anesthesia to allow awake off-pump coronary artery bypass grafting (OPCAB). In addition, we present the first published cases of awake cardiac surgery in Canada.

\section{Case 1}

A 65-yr-old man with normal ejection fraction was scheduled for awake OPCAB in March 2005. The patient had undergone stenting of the proximal left descending coronary artery in June 2004, an intra-stent occlusion was opened via percutaneous transluminal coronary angioplasty in October 2004, and additional stenting was performed in December 2004. At the time of surgery the latter stent remained patent. However, proximal to the stent, there was an $85 \%$ occlusion in addition to a $60 \%$ occlusion of the left main coronary artery. He was scheduled for a left anterior descending (LAD) artery coronary bypass graft using his left internal thoracic artery (LITA), and a venous graft to the circumflex artery. He suffered from hypertension and had stopped smoking ten years previously. He had no other comorbidity.

After approval of the local Scientific and Ethics Committee for permission to publish case series, the patient gave consent for awake cardiac surgery and was familiarized with the proposed protocol.

Prior to surgery, five-lead electrocardiography, pulse oximetry and non-invasive blood pressure monitoring were applied. A 16G-cannula was placed in the dorsum of the left hand. A high thoracic epidural catheter (TEA) was placed at the T2/3 level under local anesthesia in standard sterile fashion, with the catheter inserted at $9 \mathrm{~cm}$ from the skin. Proper placement of the catheter was tested using $4 \mathrm{~mL}$ of lidocaine $2 \%$ with adrenaline. In addition, cannulations of the right femoral artery for invasive blood pressure monitoring and right subclavian vein for central venous pressure monitoring were performed under local anesthesia.

In order to facilitate harvesting of the saphenous vein, a 3:1 block was performed after electric nerve stimulation injecting $20 \mathrm{~mL}$ of a bupivacaine $0.5 \%$ / lidocaine $2 \%$ mixture ( $1: 1$ ratio) on the left side. The installation of all catheters required less than $45 \mathrm{~min}$ and was well tolerated by the patient.

For regional anesthesia, an infusion of bupivacaine $0.5 \%$ with sufentanil $2 \mu \mathrm{g} \cdot \mathrm{mL}^{-1}$ was started at $30 \mathrm{~mL} \cdot \mathrm{h}^{-1}$ until the dermatomes $\mathrm{Tl}-8$ were blocked

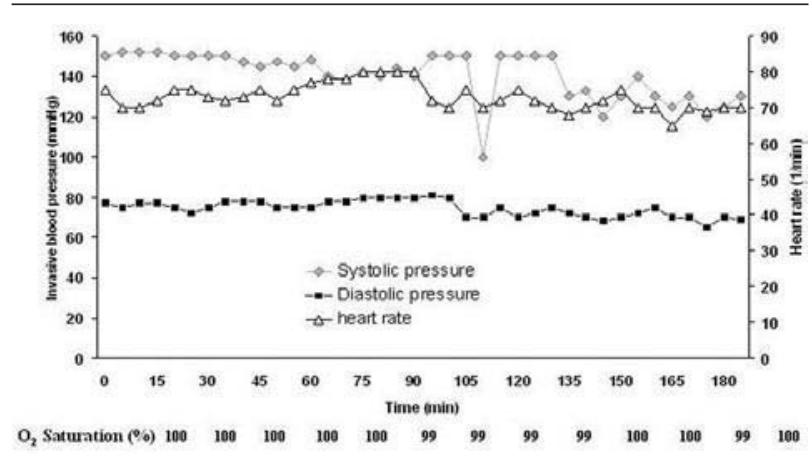

FIGURE 1 Hemodynamic variables and peripheral oxygen saturation throughout the first case.

(testing with ice; this was achieved after $10 \mathrm{~mL}$ ); then the infusion was adjusted between 4 to $7 \mathrm{~mL} \cdot \mathrm{hr}^{-1}$. Propofol 25 to $40 \mu \mathrm{g} \cdot \mathrm{kg}^{-1} \cdot \mathrm{min}^{-1}$ was infused throughout surgery to maintain an adequate response to verbal commands. In addition, oxygen was applied via a facial mask at $12 \mathrm{~L} \cdot \mathrm{min}^{-1}$.

Perioperative monitoring consisted of five-lead electrocardiography, invasive blood pressure, peripheral oxygen saturation and expired $\mathrm{CO}_{2}$ measured at the facemask. Before the start of surgery, the blood pressure was $145 / 75 \mathrm{mmHg}$, the heart rate was $70 \cdot \mathrm{min}^{-1}$, the peripheral saturation was $100 \%$.

Surgery commenced with open harvesting of the left proximal saphenous vein without any pain or movement of the patient. This took $15 \mathrm{~min}$, and was followed by conventional median sternotomy during which both pleural cavities remained intact. The surgical retractor was applied to elevate the left hemisternum for the LITA harvesting. The patient was routinely asked whether he felt any pain, which he didn't, and the sensory level of the TEA was evaluated every 15 min.

While harvesting the LITA, the left pleural space was opened, and was immediately managed by insertion of a thoracic drain, closure of the hole, and placing the thoracic drain under suction. The patient, however, did not experience any respiratory problem during this (short) period of pneumothorax. Between opening of the pleural space and retraction of the thoracic drain, five minutes elapsed during which the patient did not suffer any respiratory distress or desaturations.

Following completion of the LITA preparation, the sternal retractor was installed to expose the heart, 
and the Cor-Vasc System stabilization device manufactured by CoroNeo ${ }^{\mathrm{TM}}$ (QC, Canada) was used to assist the myocardial revascularization on the beating heart. Care was taken not to impair the cardiac output during the cardiac manipulations. Distal grafting of the LAD was accomplished within five minutes. Next, preparation and distal grafting of the circumflex artery was performed. Throughout the ischemic period, blood pressure and heart rate remained stable (Figure 1). Finally, a proximal aorto-saphenous vein anastomosis was performed using a partial occlusion clamp on a depressurized aorta (invasive blood pressure of $125 / 65 \mathrm{mmHg}$ ).

Left and right thoracic drains were inserted into both pleural cavities at the end of surgery prophylactically - as it is routine for any cardiac surgery in our hospital setting, and small drainage tubes were inserted into the pericardial and mediastinal spaces. The chest was closed using steel wires with a surgical time of two hours and $50 \mathrm{~min}$. Throughout surgery, the hemodynamic function was stable and oxygen saturation never below 99\% (Figure 1).

At the end of surgery, the epidural infusion was changed to an infusion of bupivacaine $0.065 \%$ with 3 $\mu \mathrm{g} \cdot \mathrm{mL}^{-1}$ fentanyl at an infusion rate of 6 to $10 \mathrm{~mL} \cdot \mathrm{hr}^{-1}$, and continued for two days. The patient was transferred to the postanesthesia care unit (PACU) where routine monitoring (five-lead electrocardiogram, pulse oximetry and invasive blood pressure) was continued for six hours, after which the patient was transferred to the cardiac ward. Management in the intensive care unit was not deemed necessary. The patient had minimal chest pain after surgery, and minimal leg pain which was treated with one bolus of morphine $5 \mathrm{mg} \mathrm{sc}$.

On the fourth postoperative day, a control coronary angiogram demonstrated good graft patency. On the fifth postoperative day, the patient was discharged home.

\section{Case 2}

A 65-yr-old man was scheduled for awake coronary artery bypass grafting of his LAD with LITA, and his circumflex artery with a saphenous vein. The second patient also gave consent for awake cardiac surgery and familiarized with the protocol.

The patient had a normal left ventricular ejection fraction, and $80 \%$ occlusion of the left main coronary artery. He suffered from hypertension and had ceased smoking one month prior to surgery.

In contrast to the first patient, this patient was premedicated with lorazepam $\mathrm{l} \mathrm{mg}$ sl the evening and the morning before surgery, as well as midazolam $2 \mathrm{mg} i v$ prior to insertion of the TEA. No propofol was given during surgery since the patient remained comfortable and sedated as described for the first patient. Monitoring and locoregional anesthesia was performed as described for case 1 .

After the sternotomy, the right pleural cavity was opened and a thoracic drain was inserted immediately followed by closure of the pleural cavity. Prior to preparation of the LITA, a prophylactic thoracic drain was inserted into the left pleural cavity and left in situ. Surgery was uneventful, with stable hemodynamics and respiratory function (Figure 2). This patient was also transferred to the PACU and later to the cardiac ward bypassing intensive care. He had no postoperative complications, and was discharged from hospital on the fifth postoperative day (video, available as Additional Material at www.cja-jca.org).

\section{Discussion}

We present the first published cases of awake cardiac surgery in Canada, and the first cases of awake cardiac surgery through conventional median sternotomy in North America. In addition, we present a novel anesthetic technique of combined femoral block/high TEA for awake OPCAB. This technique allows the harvesting of the proximal saphenous vein under local block.

The technique of awake cardiac surgery was first described in $2000^{13}$ and is practiced in few centres world-wide. It seems most appropriate during OPCAB in centres with vast experience with this surgical technique. We perform $>95 \%$ of all coronary artery bypass grafting as OPCAB in our hospital and have extensive experience with this technique since 1981.

Awake cardiac surgery is at an early stage of development and poses high demands on the surgical and anesthetic team. The anesthesiologist should have extensive expertise in TEA for cardiac surgery; in our hospital, > 400 cases of cardiac surgery have been performed using TEA without any complication. High epidural analgesia is still considered by some anesthesiologists as too risky for cardiac surgery. We have long used TEA in cardiac surgery, including cardiac surgery with cardiopulmonary bypass. The only recent risk calculation was published in $2000,{ }^{14}$ based on all published cases of TEA in cardiac surgery. In the Ho et al. ${ }^{14}$ calculation, the risk of epidural hematoma is calculated as 1:1,500. Including all published cases of TEA used in cardiac surgery since 1999, Djaiani et al. ${ }^{15}$ modified the risk calculation to one hematoma for 7,500 cases of insertion.

Awake cardiac surgery is seen as a logical extension of immediate extubation in the operating room, as routinely practiced in our hospital. There are important psychological considerations of this technique. 

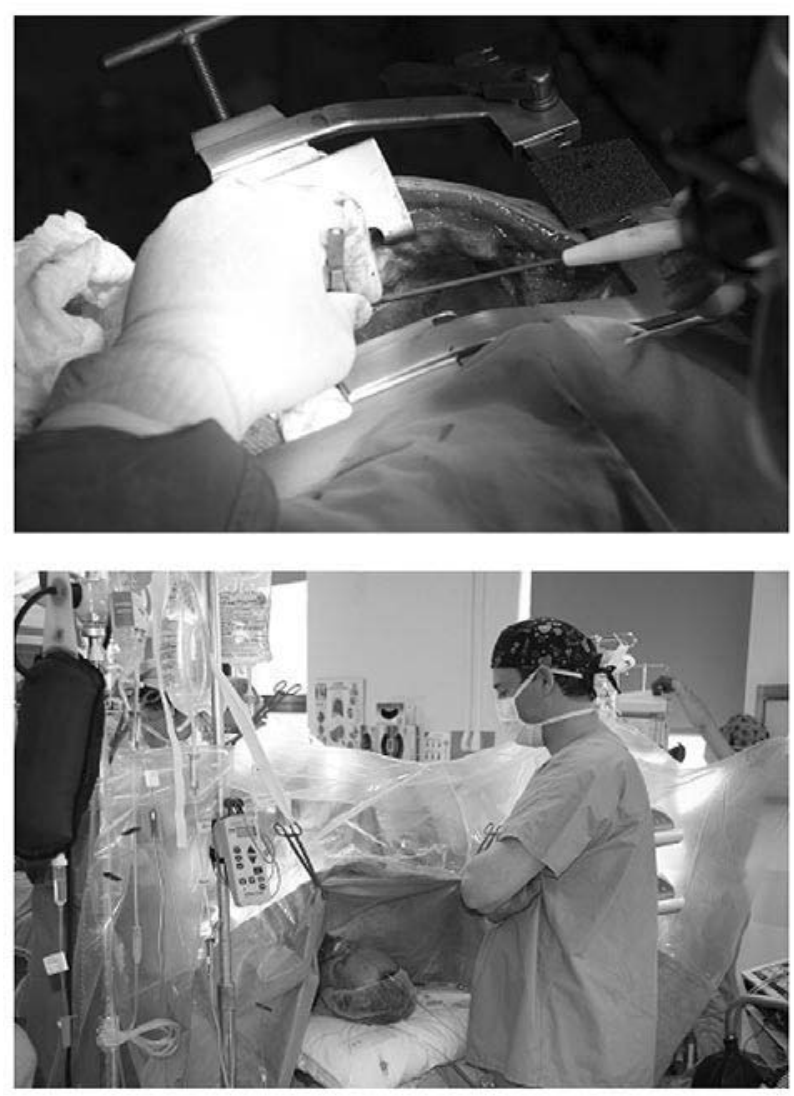

FIGURE 2 Photo of the second patient during surgery (harvesting of the mammarian artery). Note facial mask and $\mathrm{CO}_{2}$-monitoring tube for monitoring spontaneous respiration.

We carefully selected the patients before surgery, not only according to the status of their ventricular function and predicted feasibility of $\mathrm{OPCAB}$, but also according to the patient's psychological state prior to surgery. We took great time and care to explain the procedure, stressing the fact that complete analgesia of the surgical site would be ensured before any incision was attempted. In addition, we offered either a choice of music via headphones (as chosen by the first patient) or light sedation (chosen by second patient). The patients were assured that should any problem arise or if they wished, regional anesthesia would be converted immediately into general anesthesia.

Another anesthetic consideration is the fact that opening of the pleural space in a spontaneously breathing patient can happen during surgery, mostly during median sternotomy and preparation of the mammary artery. Several strategies can be used to address this complication: deliberate opening of the pleural space once accidental opening occurs without insertion of a thoracic drain or with insertion of a thoracic drain, and subsequent suction. Alternatively, temporary insertion of a thoracic drain and suction with closure of the pleural cavity and subsequent retraction of the thoracic drain (as performed in our first case), insertion of a thoracic drain and suction with closure of the pleural cavity (second case, right pleural cavity) or prophylactic insertion of the thoracic drain prior to preparation of the LITA (second case, left pleural cavity) may be considered. It is known from some cases of deliberate pneumothorax in patients undergoing awake cardiac surgery via thoracotomy, ${ }^{10}$ that unilateral pneumothorax is well tolerated throughout surgery. Another possible strategy would be the prophylactic insertion of two pleural drains prior to sternotomy, which would possibly add to the safety of the sternotomy.

Another challenge of the awake technique for OPCAB is the potential for patient movement. In both cases the patient moved his legs during periods of changing sedation levels. Immediate communication with the patient restored the tranquil operating conditions; this problem is well known to surgeons who regularly perform surgery under local or regional anesthesia, but might be new for cardiac surgeons. Great skill is required of the anesthesiologist to maintain control in ensuring a quiet and still patient. It is important to create a comfortable atmosphere for the patient - application of music, installation of monitoring devices before entering the operating theatre, and adopting appropriate communication with the patient throughout surgery.

The advantages of awake cardiac surgery are the maintenance of spontaneous respiration, possible shorter length of hospital stay due to earlier mobilization, and faster recovery of mental and physical capacity of the patient. Essentially, all arguments which favour regional anesthetic techniques are also applicable to awake cardiac surgery. However, many more cases of awake cardiac surgery are needed to truly define its advantages for the patient.

A problem with this technique is that it is only applicable for highly selected patients with good ventricular function, where there is lower risk of hypotension during manipulation of the heart, and less risk to convert to on-pump CABG. Our novel technique of femoral 3:1 block and high TEA solves the problem of vein harvesting whenever proximal (thigh) harvesting of the saphenous vein is performed.

We are at the beginning of a possible new pathway in cardiac surgery. We see this as a further step towards simplification of our technique, and towards possible outpatient cardiac surgery. It is too early to define 
the place of awake cardiac surgery within the whole of cardiac surgery, since it is has been performed only in highly selected patients with good left ventricular function. We have applied this technique in cases of two grafts, but do not believe that the number of grafts is a limiting factor as long as there is sufficient cardiac output during manipulation of the heart.

We conclude that awake OPCAB is feasible in selected patients with preserved ventricular function. The technique presents two unique concerns: unintentional opening of the pleural cavity, and possible patient movement during surgery. However, we believe that these two concerns can easily be addressed with meticulous care. Further experience is required to define the technical limitations of this technique before randomized studies should be undertaken to better define the role and safety of awake coronary artery bypass procedures in the future of cardiac surgery.

\section{References}

1 Chakravarthy $M$, Thimmangowda P, Krishnamurthy J, Nadiminti S, Jawali $V$. Thoracic epidural anesthesia in cardiac surgical patients: a prospective audit of 2,113 cases. J Cardiothorac Vasc Anesth 2005; 19: 44-8.

2 Pastor MC, Sanchez MJ, Casas MA, Mateu J, Bataller $M L$. Thoracic epidural analgesia in coronary artery bypass graft surgery: seven years' experience. J Cardiothorac Vasc Anesth 2003; 17: 154-9.

3 Kessler P, Neidhart G, Lischke V, et al. Coronary bypass operation with complete median sternotomy in awake patients with high thoracic peridural anesthesia (German). Anaesthesist 2002; 51: 533-8.

4 Aybek T, Dogan S, Neidhart G, et al. Coronary artery bypass grafting through complete sternotomy in conscious patients. Heart Surg Forum 2002; 5: 17-20.

5 Kessler P, Neidhart G, Bremerich DH, et al. High thoracic epidural anesthesia for coronary artery bypass grafting using two different surgical approaches in conscious patients. Anesth Analg 2002; 95: 791-7.

6 Aybek T, Dogan S, Kessler P, et al. Total arterial bypass operations with complete sternotomy in the awake patient (German). Z Kardiol 2002; 91: 238-42.

7 Meininger D, Neidhart G, Bremerich DH, et al. Coronary artery bypass grafting via sternotomy in conscious patients. World J Surg 2003; 27: 534-8.

8 Aybek T, Kessler P, Khan MF, et al. Operative techniques in awake coronary artery bypass grafting. J Thorac Cardiovasc Surg 2003; 125: 1394-400.

9 Anderson MB, Kwong KF, Furst AJ, Salerno TA. Thoracic epidural anesthesia for coronary bypass via left anterior thoracotomy in the conscious patient. Eur J Cardiothorac Surg 2001; 20: 415-7.
10 Anderson MB, Kwong KF, Furst AJ, Salerno TA. Thoracic epidural anesthesia for cardiac surgery via left anterior thoracotomy in the conscious patient. Heart Surg Forum 2002; 5: 105-8.

11 Gatti G, Piccione R, Pugliese P. Thoracic epidural anesthesia for off-pump coronary artery bypass grafting in a spontaneously breathing conscious patient. Ital Heart J 2003; 4: 565-7.

12 Karagoz HY, Kurtoglu M, Bakkaloglu B, Sonmez B, Cetintas T, Bayazit $K$. Coronary artery bypass grafting in the awake patient: three years' experience in 137 patients. J Thorac Cardiovasc Surg 2003; 125: 1401-4.

13 Karagoz HY, Sonmez B, Bakkaloglu B, et al. Coronary artery bypass grafting in the conscious patient without endotracheal general anesthesia. Ann Thorac Surg 2000; 70: 91-6.

14 Ho AM, Chung DC, Joynt GM. Neuraxial blockade and hematoma in cardiac surgery: estimating the risk of a rare adverse event that has not (yet) occurred. Chest 2000; 117: 551-5.

15 Djaiani G, Fedorko L, Beattie WS. Regional anesthesia in cardiac surgery: a friend or a foe? Semin Cardiothorac Vasc Anesth 2005; 9: 87-104. 